\title{
EDITORIAL
}

\section{New physics for medical physics}

\author{
This month we examine examples of how advances from different areas of physics can lead \\ to medical applications.
}

Artificial intelligence was trending this summer at the annual meeting of the American Association of Physicists in Medicine (AAPM), one of the major conferences in medical physics. The trend is unsurprising given the advances enabled by deep learning in almost every field, but it brings up the question of whether the field of medical physics still needs advances in fundamental physics.

Many of the basic technologies of medical physics are well-established, using well-understood physics principles. X-ray imaging and radiation therapy have been in use for over a century, positron emission tomography (PET) was invented in the 1950s and magnetic resonance imaging (MRI) was developed in the 1970s. Clinical applications of these technologies have improved dramatically over the years, in many cases because of improvements in engineering. But are there opportunities for physicists to develop fundamentally new approaches for medical physics technologies? There are. Here are some examples.

Owing to various factors, discussed in our March Focus issue, many nuclear research reactors are being shut down. One immediate consequence is the shortage of medical radioisotopes, such as ${ }^{99 \mathrm{~m}} \mathrm{Tc}$, which is widely used as a radioactive tracer in nuclear imaging. In a Comment in this issue, Kevin Charlton discusses alternative technologies to produce a sustainable sup-

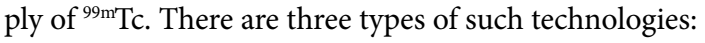

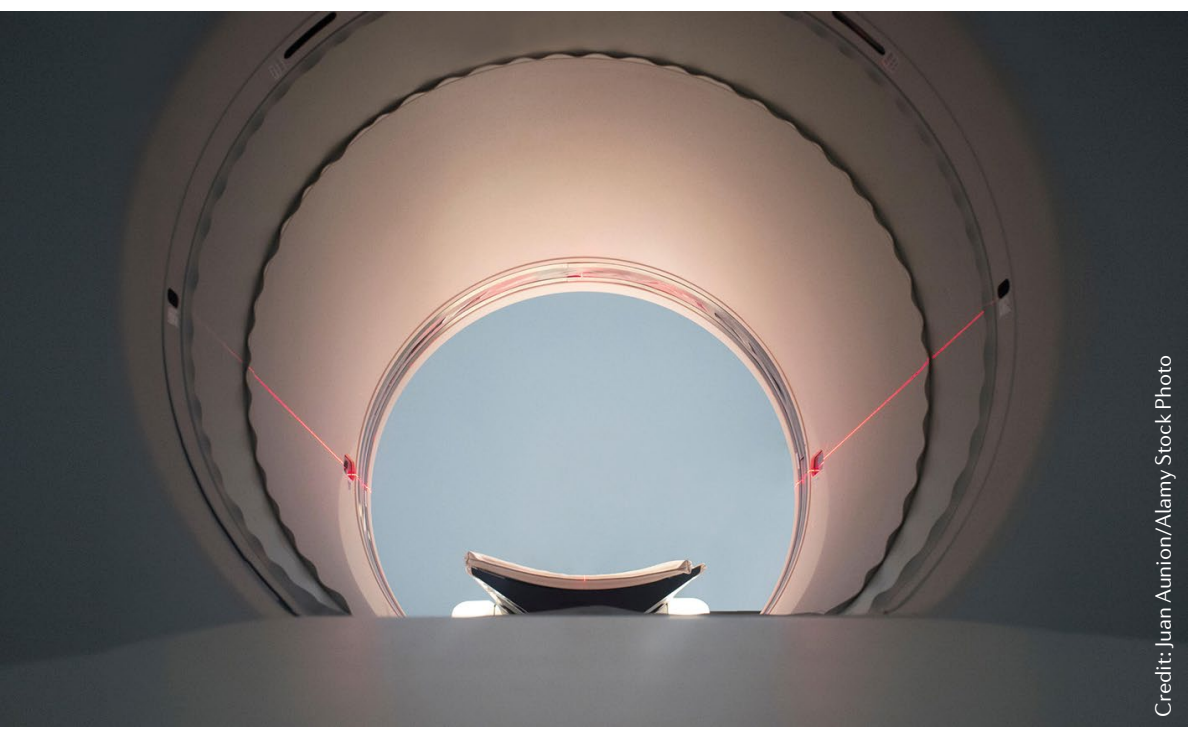

particle accelerator-based, those that use alternative uranium fission processes and new chemical separation methods. Whereas accelerator-based technologies might sound impractical given the size and cost of particle accelerators such as cyclotrons or electron accelerators, a solution may come from an unexpected place. In a Comment, Jose Alonso and colleagues discuss how a compact cyclotron designed for sterile neutrino searches can be used for the efficient production of radioisotopes such as ${ }^{68} \mathrm{Ge}$ (used to generate isotopes for PET) and ${ }^{225} \mathrm{Ac}$ (used in radiotherapy).

In medical imaging too, expertise from particle physics experiments can bring unexpected solutions. In a Comment, Paweł Moskal and colleagues describe how in PET short-lived positronium atoms are formed inside the biological tissue and tracking their decay products (an electron and a positron) can provide more information about tumours. However, putting this into practice requires a good understanding of positronium decays inside the biological tissue: an exciting opportunity for physicists, biologists and clinicians to work together.

Silicon-tracking detector technologies have developed significantly over the past 40 years thanks to advances both in semiconductor technologies for consumer electronics and research and development for particle detectors, leading to spin-off applications in other fields. Philip Allport discusses some of these developments in a Technical Review. In a Feature, Zoe Budrikis looks at one of the applications of silicon-tracking detectors outside particle physics. The Medipix chip, a by-product of the detector development for the Large Hadron Collider, enables stunning 3D colour X-ray imaging such as the one featured on this month's cover. However, as Budrikis found, getting this technology into hospitals remains a daunting task.

Another emerging direction is the application of quantum technologies in medical imaging, for example, quantum sensors based on nitrogen-vacancy centres in a diamond could enable nanoscale-resolution MRI; other types of quantum sensors could be used for non-invasive brain imaging. Such research projects benefit from European and/or national funding, so new developments can be expected in the coming years.

Whether coming from particle or quantum physics, new ideas are finding their way into medical physics applications. The road from the physics lab to doctors in hospitals is almost always long, but these new technologies are worth keeping an eye on. 